\title{
The effect of implant placement torque on crestal bone remodeling after I year of loading
}

This article was published in the following Dove Press journal:

Clinical, Cosmetic and Investigational Dentistry

\author{
Salwa Aldahlawi ${ }^{1}$ \\ Angela Demeter ${ }^{2}$ \\ Tassos Irinakis ${ }^{3}$ \\ 'Department of Basic and Clinical \\ Oral Sciences, College of Dentistry, \\ Umm Al-Qura University, Mecca, \\ Saudi Arabia; ${ }^{2}$ Private Practice, \\ Calgary, Alberta, Canada; ${ }^{3}$ Graduate \\ Periodontic Program, Faculty of \\ Dentistry, University of British \\ Columbia, Vancouver, British \\ Columbia, Canada
}

Purpose: The aim of this study was to evaluate and compare crestal bone levels (CBLs) after 1 year of loading of self-tapping bone condensing implants placed with high insertion torque (IT) compared to those placed with lower IT.

Materials and methods: A retrospective chart review of 66 consecutive patients who received at least one self-tapping bone condensing implant and were in function for at least 1 year was conducted. On the basis of intrasurgical notes documenting the implant IT, the patient population was divided into group A (implant IT, $>55 \mathrm{Ncm}$ ) and group B (IT, $<55 \mathrm{Ncm}$ ). Radiographs taken immediately after insertion and during annual follow-up appointments were evaluated for detecting crestal bone loss. The relationship between IT and crestal bone loss, bone density, and jaw location were analyzed, and a $P$-value of 0.05 was considered to be statistically significant. Results: A total of 113 self-tapping bone condensing NobelActive ${ }^{\mathrm{TM}}$ implants were placed. The average follow-up period from the placement of the implant restoration was $12.87( \pm 4.83)$ months. Six implants were classified as failures resulting in overall survival rate of $94.6 \%$. Implants in group A had a mean IT of $67.35 \pm 4.0 \mathrm{Ncm}$, whereas implants in the group B had a mean IT of $37.9 \pm 12.62 \mathrm{Ncm}$. Implants in group A had statistically significant crestal bone loss compared to implants in group B $(0.95 \pm 1.60$ and $0.18 \pm 0.68 \mathrm{~mm}$, respectively). Group A implants placed in the mandible showed significantly more pronounced crestal bone loss $(2.12$ $\pm 1.99 \mathrm{~mm})$ compared to those placed in the maxilla $(0.25 \pm 0.65 \mathrm{~mm} ; P<0.05)$; however, this was not the case in group B implants.

Conclusion: Implants inserted with high IT ( $>55 \mathrm{Ncm}$ ) showed more peri-implant bone remodeling than implants inserted with a less assertive IT $(<55 \mathrm{Ncm})$. Bone density and jaw location affect IT and CBLs.

Keywords: dental implant, nobelactive, insertion torque, torque, crestal bone level, marginal bone level

\section{Introduction}

Implant primary stability (PS) is defined as "the stability of the implant immediately post insertion". ${ }^{1}$ As it is related to implant micro motion, PS has been considered in the past to be an important prognostic factor for osseointegration and implant success. ${ }^{2}$ Implant insertion torque (IT) is frequently used as an indication of implant PS, and, although there is no consensus on what is considered the minimal torque needed for an implant to be successful, clinicians frequently use a torque value in the range of 20-40 Ncm. ${ }^{2,3}$ Many factors can affect implant IT, for example, initial implant bone contact, bone quality at the osteotomy site, ${ }^{4}$ osteotomy preparation technique, and implant geometry/morphology. ${ }^{5}$
Correspondence: Salwa Aldahlawi

Department of Basic and Clinical Ora Sciences, College of Dentistry, Umm al-Qura University. P.O. Box 7I5, Makkah, 21955

Saudi Arabia

Tel +966500559365

$\mathrm{Fax}+966125270189$

Email sadahlaawi@uqu.edu.sa 
The NobelActive ${ }^{\mathrm{TM}}$ Implant (Nobel Biocare USA, LLC, Yorba Linda, CA, USA) features a tapered body and a variable thread design with a high pitch and long wings that permit the implant to condense the surrounding bone during insertion. This potentially improves the implant stability and allows a higher IT to be reached. ${ }^{5} \mathrm{An}$ average IT of $50 \mathrm{Ncm}$ has been reported in the literature when the NobelActive system is being used. ${ }^{5,6}$ This feature makes it ideal to use in the areas where implant stability can be challenging. The higher PS is especially beneficial when it is placed immediately in fresh extraction sockets or in the areas of soft (type IV) bone. ${ }^{7,8}$ Due to its bone condensing ability, the system seems to have an advantage in thin ridges where it minimizes the need for bone grafting. ${ }^{9}$ The ability to reach high IT makes it a suitable system for immediate or early loading protocols. ${ }^{6}$ However, some risks are involved when attempting to reach high IT as jaw fracture has been reported in dense (type I) bone or in thin ridges. ${ }^{5,6}$

The effect of high IT on osseointegration and crestal bone levels (CBLs) has been a controversy dispute in the literature. Historically, excessive IT ( $>50 \mathrm{Ncm}$ ) has been assumed to compress the surrounding bone and reduce microcirculation. This may induce bone necrosis and therefore negatively affect the implant osseointegration. However, this concept has been challenged. Animal studies did not show a negative effect on osseointegration in high IT group (up to $150 \mathrm{Ncm}$ ), ${ }^{10,11}$ whereas human studies showed controversial results. ${ }^{12-14} \mathrm{~A}$ recent systematic review indicated that there is no significant difference in bone resorption and implant failure rate between implants placed with high IT (up to $176 \mathrm{Ncm}$ ) and low (30 $\mathrm{Ncm}){ }^{15}$ However, the authors reported that most studies have a high risk of bias. Recently, significant marginal bone loss and buccal soft tissue recession were reported in implants placed with high IT $(>50 \mathrm{Ncm})$ compared to those placed with regular IT $(<50 \mathrm{Ncm})$ at 1 - and 3-year post-loading follow-up..$^{14,16}$

This study was conducted to evaluate and compare CBLs after 1 year of loading of self-tapping bone condensing NobelActive implants placed with high IT compared to those placed with lower IT. The null hypothesis is that IT does not affect the CBL at short-term follow-up.

\section{Materials and methods Study design}

This study is a retrospective chart review of consecutive patients who received at least one NobelActive implant and treated by one of two experienced periodontists in a private practice setting. Human ethics approval was attained from the Clinical Research Ethics Board, University of British Columbia, Office of Research Services. All patients provided a written informed consent to review their medical records as per University of British Columbia policy. The inclusion criteria for the study were as follows: patients who received at least one NobelActive implant that had been in function for an average post-loading period of 12 months and availability of post-loading radiographic and clinical follow-up assessments with radiographs depicting implants in their entirety with clearly discernible threads, thus making them radiographically diagnostic.

The implants were placed in partially dentate and edentulous sites of the maxilla and mandible, in either native bone or at sites augmented previously or simultaneously with bone grafting. Implants were immediately placed in fresh extraction sockets or in native bone and were restored with immediate or delayed loading protocols.

\section{Surgical protocol}

All procedures were done under local anesthesia. All surgeries were done under antibiotic prophylaxis with $2 \mathrm{~g}$ amoxicillin (or $600 \mathrm{mg}$ clindamycin in penicillin-allergic patients). Postoperatively, patients took $500 \mathrm{mg}$ amoxicillin (or 300 $\mathrm{mg}$ clindamycin) three times daily for 7 days. Patients were asked to rinse with $0.2 \%$ chlorhexidine gluconate mouthwash for 1 minute pre-operatively and then twice a day for up to 2 weeks post-operatively. Appropriate pain medication was prescribed after the implant installation surgery. All procedures were done following the NobelActive original procedural protocol, which advocated the placement to 70 $\mathrm{Ncm}$. A surgical guide was used to prosthetically determine the implant position. Patients were seen at 10-14 days postoperatively for a follow-up and suture removal. All patients were referred to the restorative dentist for final restoration(s), unless immediate loading was performed in which case the restorative clinician was present during the surgery. Patients were recalled at regular intervals for periodontal and implant maintenance.

\section{Data collection}

Patient charts were audited for patients' demographic data and smoking and medical history. Intra-surgical notes documenting implant width, length, location, IT, and bone density were recorded. The IT was measured using the NobelActive hand torque wrench instrument from the NobelActive surgery kit (Figure 1). Implants were divided into two groups based on the IT value; group A with IT $>55 \mathrm{Ncm}$ and group B with IT $\leq 55 \mathrm{Ncm}$. Bone density was recorded as soft, medium, and 

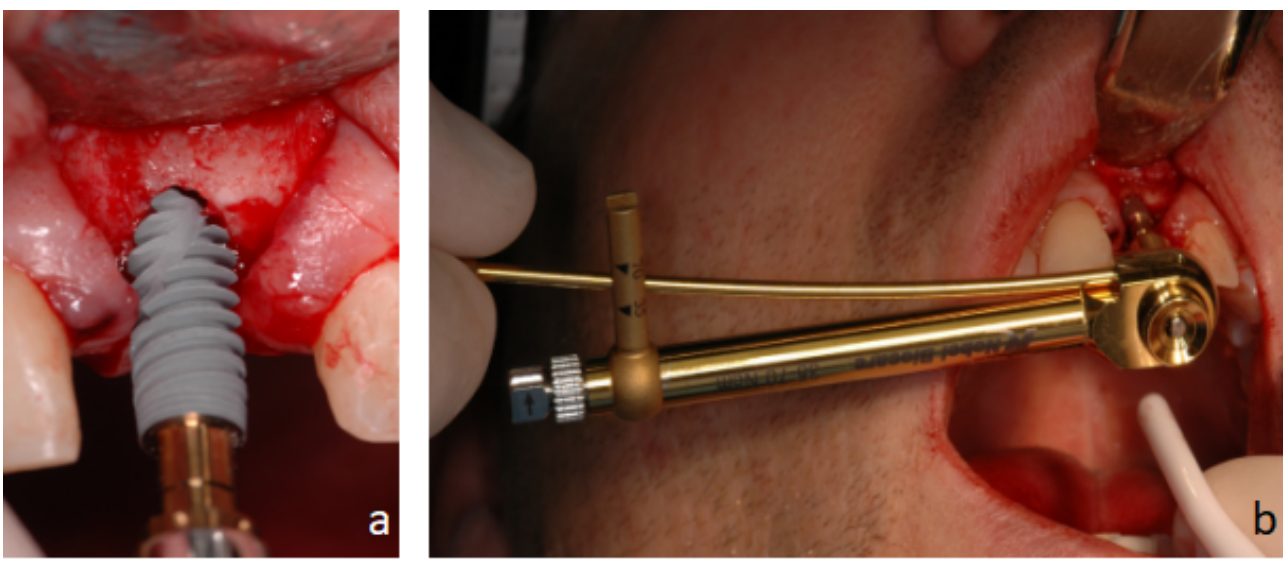

Figure I The NobelActive ${ }^{\mathrm{TM}}$ implant features tapered body and accentuated threads/wings.

Notes: (A) The osteotomy site is slightly underprepared, which allows for the surrounding bone to be compressed laterally during implant insertion. (B) The manual torque wrench for NobelActive system allows controlled measurement of insertion torque up to $70 \mathrm{Ncm}$.

dense. Dense bone corresponded to type I bone and soft bone correspond to type IV. Medium bone density corresponded to type II and III, which are difficult to differentiate clinically. ${ }^{7}$

Procedural information regarding the timing of implant placement (in extraction socket or healed ridges), the need for bone augmentation (simultaneous with implant placement or staged), and loading protocol (immediate loading or delayed) were collected.

Intraoral radiographs taken at implant placement and during follow-up appointments were scanned and measured by Romexis 2.2.7R Software System (Planmeca, Helsinki, Finland). The coronal margin of the implant collar and the most coronal aspect of the bone-to-implant contact were used as reference points for the linear measurements of marginal bone loss. Known implant lengths were used for the calibration of the measurements. Measurements of the mesial and distal CBLs adjacent to each implant were performed. The amount of true bone resorption and the difference between the initial bone level and the bone level at follow-up examinations were calculated (Figure 2). For those implants placed intentionally in a supra-crestal position, the height of the implant collar above the alveolar crest during placement was subtracted from follow-up radiographs. The mesial, distal, average, and worst marginal bone loss values were recorded for each implant. One examiner who was not involved in the treatment of patients evaluated all intraoral radiographs. Intra-examiner reliability was assessed in the sample of 25 randomly selected periapical radiographs that were measured twice, once at baseline and again after 4 months. The two recordings were highly correlated with a Pearson correlation coefficient of $0.981(P=0.000)$ and $0.994(P=0.000)$ at the mesial and distal measurements, respectively.

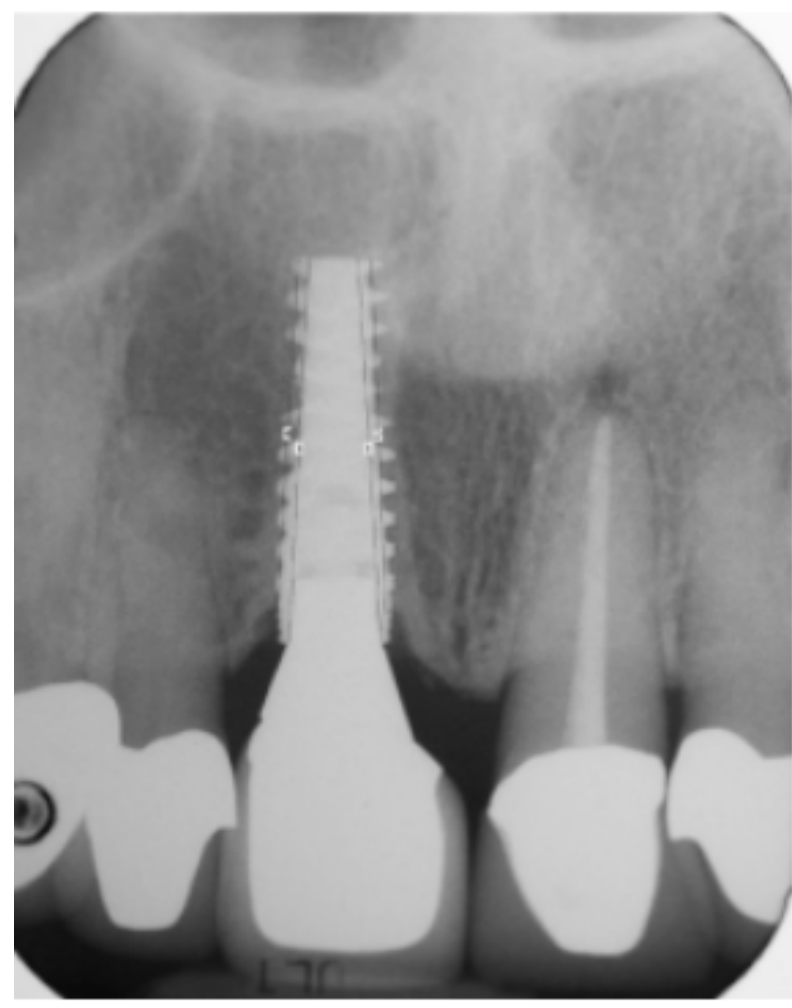

Figure 2 Example of measurements taken to estimate the crestal bone level. Notes: The coronal margin of the implant collar and the most coronal aspect of the bone-to-implant contact were used as reference points for the linear measurements of crestal bone loss. The known implant length was used to compensate for radiograph magnification or angulation. Measurements were done on the mesial and distal aspects.

Statistical analysis was completed using the PASW statistics program (version 18.0, PASW, Chicago, IL, USA). Descriptive statistics were used for all evaluated parameters, and baseline patient and implant characteristics were summarized in terms of frequencies and percentages. Implant 
IT, jaw location, and CBL were compared by independent $t$-test. One-way ANOVA was used to compare bone density, implant IT, and CBL. The $P$-value of 0.05 was considered to be statistically significant.

\section{Results}

A total of 66 patients (31 females and 35 males) were included in the study. The average age of patients at the time of implant placement was of $56.31( \pm 16.20)$ years. A total of 10 subjects reported to be smokers. The demographic data are shown in Table 1.

Most subjects (62\%) received one implant; $21 \%$ received two implants, $9 \%$ received three implants, and $6 \%$ received more than three implants. A total of 113 NobleActive implants were placed. Group A had 51 implants, whereas group B had 62 implants.

Guided bone regeneration (GBR) or sinus augmentation was performed prior to the placement of 12 implants (10.6\%). Average healing period of sites was 7.2 months (5-9

Table I Subject's demographic data and implants distribution

\begin{tabular}{|l|l|l|}
\hline & Group A & Group B \\
\hline Number of subjects & 31 & 44 \\
\hline Age (years) & 61 & $5 \mathrm{I}$ \\
\hline Age range (years) & $28-80$ & $29-84$ \\
\hline Gender (male/female) & $16 / 15$ & $22 / 22$ \\
\hline Smoker & 5 & 5 \\
\hline Insertion torque (mean \pm SD) & $67.35 \pm 4.0 \mathrm{Ncm}$ & $37.9 \pm 12.62 \mathrm{Ncm}$ \\
\hline Number of implants & $5 \mathrm{I}$ & 62 \\
\hline Location & $3 \mathrm{I}$ & 53 \\
\hline Maxilla & 20 & 9 \\
\hline Mandible & \multicolumn{2}{|l|}{} \\
\hline Bone augmentation & 23 & 22 \\
\hline Simultaneous bone augmentation & 5 & 7 \\
\hline Prior bone augmentation & 5 & 20 \\
\hline Immediate placement & 19 & 9 \\
\hline Immediate loading & 9 & 5 \\
\hline Immediate placement + loading & 7 &
\end{tabular}

months) prior to implant placement. Simultaneous GBR or sinus augmentation was performed in 45 of the 113 implants (39.8\%). The most common bone graft material used was Bio-Oss ${ }^{\circledR}$ (Geistlich Pharma North America Inc., Princeton, NJ, USA), which is used in $41 \%(n=23)$ of sites, followed by Puros ${ }^{\circledR}$ Allograft (Zimmer Biomet, Warsaw, IN, USA) in $30 \%(n=17)$ of the sites and DynaBlast ${ }^{\mathrm{TM}}$ (Citagenix, Laval, QC, Canada) in $10.7 \%$ of sites $(n=6)$.

A one-stage implant protocol was employed for $63.7 \%$ $(\mathrm{n}=72)$ of implants and a two-stage protocol for $36.3 \%$ $(n=41)$ of the implants. Of the evaluated implants, $34.5 \%$ were placed immediately in fresh extraction sockets $(n=39)$, $15.9 \%$ were immediately loaded $(n=18)$, while $10.6 \%$ were both placed immediately into fresh extraction sockets and immediately loaded $(n=12)$.

Implants in group A had a mean IT of $67.35 \pm 4.0 \mathrm{Ncm}$, whereas implants in the group B had a mean IT of $37.9 \pm$ $12.62 \mathrm{Ncm}$. Implants placed immediately into extraction sockets had a mean IT of $53.4 \pm 15.3 \mathrm{Ncm}$, while implants that were immediately loaded had a mean IT of $54.4 \pm 14.2$ Ncm. Implants that were both immediately placed and loaded had a mean IT of $58.3 \pm 13.5 \mathrm{Ncm}$. (Table 1)

\section{Implant survival rates}

A total of 113 implants were placed. Six implants were classified as failures (5.4\%) due to implant mobility, progressive bone loss, or infection. The overall survival rate was $94.6 \%$.

Two failures in group A occurred after 3 and 5 months of implant placement. The four failures in group B occurred between 1 and 11 months after implant placement. None of the subjects who experienced failure were smokers. Five of the six failures occurred prior to implant restoration and one failure occurred 11 months after immediate loading. Description of failures is presented in Table 2. This resulted in survival rates of $96 \%$ and $93.5 \%$ in group A and group B, respectively.

Table 2 Data on failed implants

\begin{tabular}{|c|c|c|c|c|c|c|}
\hline Site & $\begin{array}{l}\text { Torque } \\
(\mathrm{Ncm})\end{array}$ & $\begin{array}{l}\text { Smoking } \\
\text { status }\end{array}$ & $\begin{array}{l}\text { Time from placement to } \\
\text { removal (months) }\end{array}$ & Bone augmentation & Placement & Immediate loading \\
\hline$\# 36$ & 70 & No & 5 & $\begin{array}{l}\text { Simultaneous with implant } \\
\text { placement }\end{array}$ & Conventional & Conventional \\
\hline$\# 24$ & 70 & No & 3 & None & Immediate & Conventional \\
\hline$\# 25$ & 20 & No & 3 & $\begin{array}{l}\text { Simultaneous with implant } \\
\text { placement }\end{array}$ & Immediate & Conventional \\
\hline$\# 35$ & 50 & No & I & Prior to implant placement & Conventional & Conventional \\
\hline$\# 44$ & 50 & No & 11 & None & Immediate & Immediate \\
\hline$\# 22$ & 40 & No & 5 & None & Immediate & Conventional \\
\hline
\end{tabular}




\section{Mean crestal bone loss}

The average follow-up period from the placement of the implant restoration was $12.87( \pm 4.83)$ months with the range of 4-27 months.

A total of $79.6 \%$ of the implants placed during the study period had crestal bone loss of $<1.0 \mathrm{~mm}, 2.65 \%$ had crestal bone loss of $1-2 \mathrm{~mm}, 8 \%$ had crestal bone loss of $2-4 \mathrm{~mm}$, and $4.4 \%$ had crestal bone loss of $>4 \mathrm{~mm}$.

The mean mesial crestal bone loss $( \pm \mathrm{SD})$ was $0.52( \pm 1.28)$ $\mathrm{mm}$ with a minimum of $0 \mathrm{~mm}$ and a maximum of $6.1 \mathrm{~mm}$. The mean distal crestal bone loss $( \pm \mathrm{SD})$ was $0.54( \pm 1.33) \mathrm{mm}$ with a minimum of $0 \mathrm{~mm}$ and a maximum of $5.2 \mathrm{~mm}$. Since the mean mesial and distal crestal bone loss measurements were highly correlated (Pearson correlation coefficient $=0.643$, $P=0.000)$, the average mean crestal bone loss $(0.53 \pm 1.25$ $\mathrm{mm}$ ) was used for subsequent statistical analysis (minimum, $0 \mathrm{~mm}$; maximum, $5.6 \mathrm{~mm}$; Figures 3 and 4).

\section{IT and bone loss}

Implants in group A had an average crestal bone loss of 0.95 $\pm 1.60 \mathrm{~mm}$, whereas implants in group B had an average bone loss of $0.18 \pm 0.68 \mathrm{~mm}$. A statistically significant impact of torque on crestal bone loss was observed $(P=0.001)$.

IT was highly correlated with bone density $(P=0.000)$. The mean torque was $65.0 \pm 9.7 \mathrm{Ncm}$ in dense bone, $47.8 \pm 16.6$ $\mathrm{Ncm}$ in medium bone, and $47.9 \pm 19.6 \mathrm{Ncm}$ in soft bone. More specifically, an analysis with multiple comparisons revealed a significant difference in torque values between dense and medium bone $(P=0.000)$ and dense and soft bone $(P=0.007)$. Mean marginal bone loss $( \pm \mathrm{SD})$ was $1.25( \pm 1.70) \mathrm{mm}$ for dense bone, $0.44( \pm 1.12) \mathrm{mm}$ for medium bone, and $0.86( \pm 0.65)$ $\mathrm{mm}$ for soft bone. There was a statistically significant effect of bone density on mean marginal bone loss $(P=0.004)$. Post-hoc Tukey honest significant difference test showed a statistically significant difference between dense bone and medium bone $(P=0.01)$ and between dense bone and soft bone $(P=0.004)$. However, the difference between medium and soft bone was not statistically significant $(P=0.45)$.

Group A included 32 (62.7\%) implants placed in the maxilla and 19 implants (37\%) placed in the mandible. Group B included 53 implants (85\%) that were placed in the maxilla, while the remaining implants $(n=9)$ were inserted in the mandible.

In group A, the average IT was 66 and $70 \mathrm{Ncm}$ in the maxilla and mandible, respectively. The average bone loss was 0.25 $( \pm 0.68) \mathrm{mm}$ in the maxilla and $2.12( \pm 1.99) \mathrm{mm}$ in the mandible. Greater bone loss was present in the mandible than in the maxilla, and this finding was statistically significant $(P=0.00)$.

In group B, the average IT was 36 and $47 \mathrm{Ncm}$ in the maxilla and mandible, respectively. The average bone loss was $0.19( \pm 0.65) \mathrm{mm}$ in the maxilla and $0.15( \pm 0.82) \mathrm{mm}$ in the mandible. However, this difference was not statistically significant $(P=0.10)$.
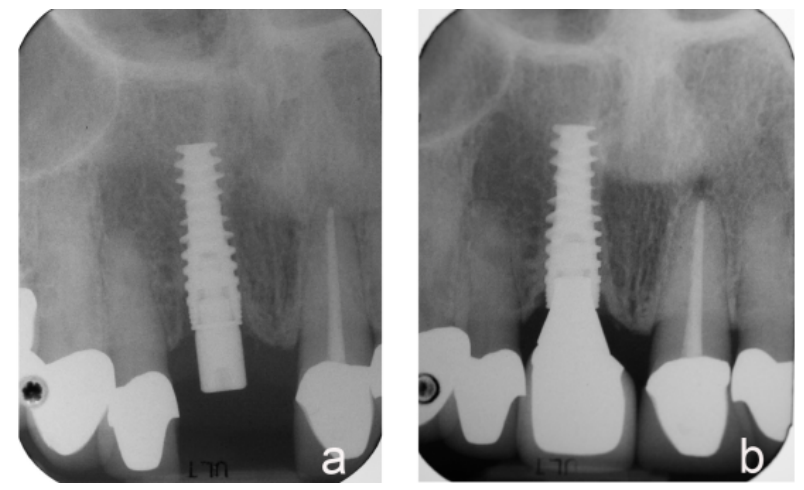

Figure 4 Periapical radiographs depicting crestal bone loss. Notes: (A) Periapical radiograph taken at the time of implant placement and (B) at I-year follow-up appointment. Crestal bone loss is observed on the mesial and distal aspects of the implant.
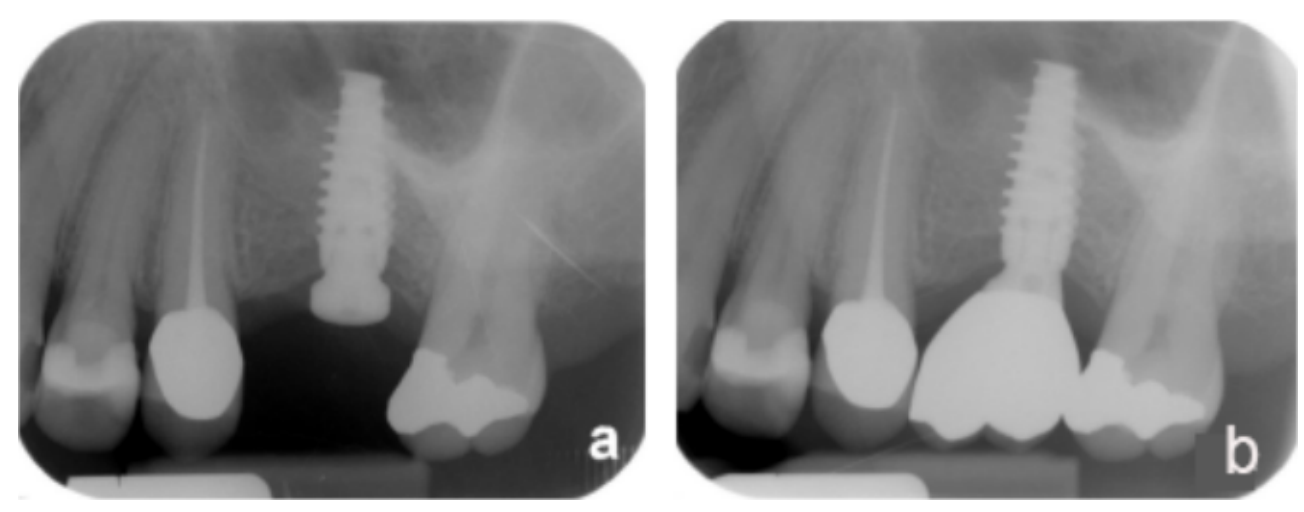

Figure 3 Periapical radiographs depicting no crestal bone loss.

Note: (A) Periapical radiograph taken at the time of implant placement and (B) at I-year follow-up appointment. 


\section{Discussion}

This study compared the changes in CBL of self-tapping bone condensing implants placed with high IT ( $>55 \mathrm{Ncm})$ and regular IT $(<55 \mathrm{Ncm})$ after 1 year of function. Moreover, the effect of bone quality and jaw location on IT and crestal bone loss was assessed.

The cumulative survival rate of the implants in this study was $94.6 \%$ with an average mean bone loss after 1 year of function of $0.53 \pm 1.25 \mathrm{~mm}$. This is comparable to marginal bone loss measurements reported by short-term reports. ${ }^{6,17}$ Also, the average marginal bone loss reported in this study was within the acceptable threshold recommended by Albrektsson and Zarb ${ }^{17}$ for rough surface implants.

The main outcomes of this study showed that NobelActive implants placed with high IT had an average crestal bone loss of $0.95 \pm 1.60 \mathrm{~mm}$, and this was significantly more pronounced when compared to crestal bone loss of implants placed with regular IT $(0.18 \pm 0.68 \mathrm{~mm})$. Frequently, implants placed in the mandible have a higher IT compared to the maxilla (70 vs $66 \mathrm{Ncm}$ in group A and $47 \mathrm{vs} 36 \mathrm{Ncm}$ in group B). The 1-year follow-up showed significantly increased crestal bone loss in implants placed in the mandible in group A (high IT) compared to the implants placed in the maxilla. This difference was not detected between the mandible and maxilla in group B (regular IT) implants. High IT was consistently achieved in dense bone compared to soft or medium bone, and subsequently, more pronounced crestal bone loss was detected in implants placed in dense bone.

Those results are consistent with the recent work of Marconcini et $\mathrm{a}^{14}$ in which implants placed with high IT ( $>50 \mathrm{Ncm}$ ) exhibited invariably significant crestal bone loss and soft tissue recession compared to implants placed with regular IT at 3-year follow-up. A similar trend was detected in the earlier follow-up of the same group of implants at 1 and 2 years. ${ }^{16,18}$ Similar to our findings, more crestal bone loss was detected in implants placed in the mandible compared to the maxilla with the average mandibular crestal bone loss in the high IT group being $1.23 \pm 0.36 \mathrm{~mm}$ compared to $0.88 \pm$ $0.43 \mathrm{~mm}$ in the maxilla. ${ }^{16}$ It is interesting that in our study, the difference between jaw location was not detected in group B (regular IT). This could be attributed to the small number of mandibular implants in this group. It could also indicate that lower IT values do not lead to the pronounced crestal bone loss in a dense bone environment (typical for the mandible).

A recent systematic review concluded that IT does not have a detrimental effect on crestal bone loss or implant failure. ${ }^{15}$ The review included only four human studies that specifically addressed CBL changes in relation to IT. Two of the studies included did not have a proper randomization to their subjects and therefore selection bias could affect their results. ${ }^{15}$ In addition, the studies had a wide range of IT (>25-176 Ncm), and there was no consensus on what was considered a high IT. Therefore, no agreement was reached on what is considered a proper IT value or on the threshold of IT that could eventfully lead to bone resorption.

Many studies confirmed the relationship between bone quality and IT. ${ }^{19,20}$ In our study, there was a consistently high IT in dense bone compared to soft bone. This was reflected in CBL changes as implants placed in dense bone had significant crestal bone loss compared to those placed in medium or soft bone. ${ }^{21}$

NobleActive implants have a geometrical structure that allows them to achieve a higher IT and PS. IT $\mathrm{s}$ of $>40$ $\mathrm{Ncm}$ have routinely been reported in the literature with this system, ${ }^{6,7,22}$ which is advantageous in areas where implant stability is challenging. In comparison with other implants systems, NobleActive drilling protocol creates an osteotomy site that is slightly underprepared, ie, smaller than the planned implant size. This allows the bone to be compressed laterally during implant insertion and therefore generates a higher IT. This is a similar concept to the use of osteotome to condense bone suggested by Summers. ${ }^{23}$ Another feature of this implant system is its ability to reach a higher IT without the risk of damaging the internal hex connection or inducing pressure necrosis. ${ }^{7,24}$ However, insertion of the implant should be performed slowly especially in the areas of dense bone, with frequent reversing to allow the cutting of bone trabeculae and reducing the insertion pressure to avoid surgical complication like bone fracture ${ }^{6}$ or crestal bone loss.

There are several limitations to this study. The retrospective nature affects the homogeneity of the sample, and every effort was done to keep the procedure and the documentation consistent, but there was no calibration among the surgeons, and clinical judgment was used for the best of the patient, which may affect the results. Other limitations include the small population, lack of comparison group, and the short-term follow-up, which make the detection of correlations between contributing factors and failure rates difficult. More studies with larger sample size and longer follow-up are needed.

\section{Conclusion}

The findings of this study showed that the self-tapping bone condensing NobelActive implants inserted with high IT ( $>55$ $\mathrm{Ncm}$ ) result in statistically significant more crestal bone loss after 1 year of loading compared to those inserted with a lower IT. Moreover, more pronounced bone loss was detected in the 
mandible when the IT was high. Although clinical outcomes for the NobelActive implant system are favorable compared to other clinically validated implant systems, clinical judgment is still needed to prevent overcompression of the surrounding bone especially in the areas of high bone density to avoid short-term unfavorable effects on CBLs.

\section{Author contributions}

SA: data acquisition, analysis and interpretation, manuscript writing, and available literature review. AD: data acquisition and analysis. TI: study design and revising the manuscript critically for important intellectual content. All authors contributed toward data analysis, drafting and revising the paper and agree to be accountable for all aspects of the work.

\section{Disclosure}

The authors report no conflicts of interest in this work.

\section{References}

1. Li H, Liang Y, Zheng Q. Meta-Analysis of Correlations Between Marginal Bone Resorption and High Insertion Torque of Dental Implants. Int J Oral Maxillofac Implants. 2015;30(4):767-772.

2. Greenstein G, Cavallaro J. Implant Insertion Torque: Its Role in Achieving Primary Stability of Restorable Dental Implants. Compend Contin Educ Dent. 2017;38(2):88-95.

3. Benic GI, Mir-Mari J, Hämmerle CH. Loading protocols for singleimplant crowns: a systematic review and meta-analysis. Int J Oral Maxillofac Implants. 2014;29 Suppl:222-238.

4. Trisi P, De Benedittis S, Perfetti G, Berardi D, Stability P. Primary stability, insertion torque and bone density of cylindric implant ad modum Branemark: is there a relationship? An in vitro study. Clin Oral Implants Res. 2011;22(5):567-570.

5. Irinakis T, Wiebe C. Clinical evaluation of the NobelActive implant system: a case series of 107 consecutively placed implants and a review of the implant features. J Oral Implantol. 2009;35(6):283-288.

6. Ho DS, Yeung SC, Zee KY, Curtis B, Hell P, Tumuluri V. Clinical and radiographic evaluation of NobelActive(TM) dental implants. Clin Oral Implants Res. 2013;24(3):297-304.

7. Irinakis $\mathrm{T}$, Wiebe $\mathrm{C}$. Initial torque stability of a new bone condensing dental implant. A cohort study of 140 consecutively placed implants. J Oral Implantol. 2009;35(6):277-282.

8. Bell C, Bell RE. Immediate restoration of NobelActive implants placed into fresh extraction sites in the anterior maxilla. J Oral Implantol. 2014;40(4):455-458.
9. Albanese M, Ricciardi G, Donadello D, et al. Alveolar splitting with piezosurgery, bone bank grafts and Nobelactive implants as an alternative to major bone grafting for maxillary reconstruction. Minerva Stomatol. Epub 2017 Jul 4.

10. Trisi P, Todisco M, Consolo U, Travaglini D. High versus low implant insertion torque: a histologic, histomorphometric, and biomechanical study in the sheep mandible. Int J Oral Maxillofac Implants. 2011;26(4):837-849.

11. Consolo U, Travaglini D, Todisco M, Trisi P, Galli S. Histologic and biomechanical evaluation of the effects of implant insertion torque on peri-implant bone healing. J Craniofac Surg. 2013;24(3):860-865.

12. Khayat PG, Arnal HM, Tourbah BI, Sennerby L. Clinical outcome of dental implants placed with high insertion torques (up to $176 \mathrm{Ncm}$ ). Clin Implant Dent Relat Res. 2013;15(2):227-233.

13. Norton MR. The influence of insertion torque on the survival of immediately placed and restored single-tooth implants. Int J Oral Maxillofac Implants. 2011;26(6):1333-1343.

14. Marconcini S, Giammarinaro E, Toti P, Alfonsi F, Covani U, Barone A. Longitudinal analysis on the effect of insertion torque on delayed single implants: A 3-year randomized clinical study. Clin Implant Dent Relat Res. 2018;20(3):322-332.

15. Berardini M, Trisi P, Sinjari B, Rutjes AW, Caputi S. The Effects of High Insertion Torque Versus Low Insertion Torque on Marginal Bone Resorption and Implant Failure Rates: A Systematic Review With Meta-Analyses. Implant Dent. 2016;25(4):532-540.

16. Barone A, Alfonsi F, Derchi G, et al. The Effect of Insertion Torque on the Clinical Outcome of Single Implants: A Randomized Clinical Trial. Clin Implant Dent Relat Res. 2016;18(3):588-600.

17. Albrektsson T, Zarb GA. Current interpretations of the osseointegrated response: clinical significance. Int J Prosthodont. 1993;6(2):95-105.

18. Alfonsi F, Borgia V, Barbato L, et al. The clinical effects of insertion torque for implants placed in healed ridges: A two-year randomized controlled clinical trial. J Oral Science \& Rehabilitation. 2016;2(4):62-73.

19. Karl M, Grobecker-Karl T. Effect of bone quality, implant design, and surgical technique on primary implant stability. Quintessence Int. Epub 2018 Jan 22:189-198.

20. Marquezan M, Osório A, Sant'anna E, Souza MM, Maia L. Does bone mineral density influence the primary stability of dental implants? A systematic review. Clin Oral Implants Res. 2012;23(7):767-774.

21. Ibañez C, Catena A, Galindo-Moreno P, Noguerol B, Magán-Fernández A, Mesa F. Relationship Between Long-Term Marginal Bone Loss and Bone Quality, Implant Width, and Surface. Int J Oral Maxillofac Implants. 2016;31(2):398-405.

22. Kielbassa AM, Martinez-de Fuentes R, Goldstein M, et al. Randomized controlled trial comparing a variable-thread novel tapered and a standard tapered implant: interim one-year results. J Prosthet Dent. 2009;101(5):293-305.

23. Summers RB. A new concept in maxillary implant surgery: the osteotome technique. Compendium. 1994;15(2):152.

24. Orentlicher G, Teich M. Evolving implant design. The NobelActive implant: discussion and case presentations. Compend Contin Educ Dent. 2010;31(1):66-70, 72-77.
Clinical, Cosmetic and Investigational Dentistry

\section{Publish your work in this journal}

Clinical, Cosmetic and Investigational Dentistry is an international, peer-reviewed, open access, online journal focusing on the latest clinical and experimental research in dentistry with specific emphasis on cosmetic interventions. Innovative developments in dental materials, techniques and devices that improve outcomes and patient satisfac-

\section{Dovepress}

tion and preference will be highlighted. The manuscript management system is completely online and includes a very quick and fair peerreview system, which is all easy to use. Visit http://www.dovepress. com/testimonials.php to read real quotes from published authors. 